\title{
MARTIN'S CONJECTURE, ARITHMETIC EQUIVALENCE, AND COUNTABLE BOREL EQUIVALENCE RELATIONS
}

\author{
ANDREW MARKS, THEODORE SLAMAN, AND JOHN STEEL
}

\begin{abstract}
There is a fascinating interplay and overlap between recursion theory and descriptive set theory. A particularly beautiful source of such interaction has been Martin's conjecture on Turing invariant functions. This longstanding open problem in recursion theory has connected to many problems in descriptive set theory, particularly in the theory of countable Borel equivalence relations.

In this paper, we shall give an overview of some work that has been done on Martin's conjecture, and applications that it has had in descriptive set theory. We will present a long unpublished result of Slaman and Steel that arithmetic equivalence is a universal countable Borel equivalence relation. This theorem has interesting corollaries for the theory of universal countable Borel equivalence relations in general. We end with some open problems, and directions for future research.
\end{abstract}

\section{INTRODUCTION}

1.1. Martin's conjecture. Martin's conjecture on Turing invariant functions is one of the oldest and deepest open problems on the global structure of the Turing degrees. Inspired by Sacks' question on the existence of a degree-invariant solution to Post's problem [21, Martin made a sweeping conjecture that says in essence, the only nontrivial definable Turing invariant functions are the Turing jump and its iterates through the transfinite.

Our basic references for descriptive set theory and effective descriptive set theory are the books of Kechris [14] and Sacks 22]. Let $\leq_{T}$ be Turing reducibility on the Cantor space $2^{\omega}$, and let $\equiv_{T}$ be Turing equivalence. Given $x \in 2^{\omega}$, let $x^{\prime}$ be the Turing jump of $x$. The Turing degree of a real $x \in 2^{\omega}$ is the $\equiv_{T}$ equivalence class of $x$. A Turing invariant function is a function $f: 2^{\omega} \rightarrow 2^{\omega}$ such that for all reals $x, y \in 2^{\omega}$, if $x \equiv_{T} y$, then $f(x) \equiv_{T} f(y)$. The Turing invariant functions are those which induce functions on the Turing degrees.

With the axiom of choice, we can construct many pathological Turing invariant functions. Martin's conjecture is set in the context of $\mathrm{ZF}+\mathrm{DC}+\mathrm{AD}$, where $\mathrm{AD}$ is the axiom of determinacy. We assume $\mathrm{ZF}+\mathrm{DC}+\mathrm{AD}$ for the rest of this section. The results we will discuss all "localize" so that the assumption of AD essentially amounts to studying definable functions assuming definable determinacy, for instance, Borel functions using Borel determinacy.

To state Martin's conjecture, we need to recall the notion of Martin measure. A Turing cone is a set of the form $\left\{x: x \geq_{T} y\right\}$. The real $y$ is said to be the base of

The results in Section 3 form part of the $\mathrm{PhD}$ thesis of the first author, which was done under the supervision of Ted Slaman. The first author would also like to thank Ben Miller for helpful conversations. The research of the second author was partially supported by NSF award DMS1001551. The research of the third author was partially supported by NSF award DMS-0855692. 
the cone $\left\{x: x \geq_{T} y\right\}$. A Turing invariant set is a set $A \subseteq 2^{\omega}$ that is closed under Turing equivalence: for all $x, y \in 2^{\omega}$, if $x \in A$ and $x \equiv_{T} y$, then $y \in A$. Martin has shown that under $\mathrm{AD}$, every Turing invariant set $A$ either contains a Turing cone, or is disjoint from a Turing cone 19. Note that the intersection of countably many cones contains a cone; the intersection of the cones with bases $\left\{y_{i}\right\}_{i \in \omega}$ contains the cone whose base is the join of the $y_{i}$. Hence, under $\mathrm{AD}$, the function

$$
\mu(A)= \begin{cases}1 & \text { if } A \text { contains a Turing cone } \\ 0 & \text { if the complement of } A \text { contains a Turing cone }\end{cases}
$$

is a measure on the $\sigma$-algebra of Turing invariant sets. This measure is called Martin measure. For the rest of this section, by a.e. we will mean almost everywhere with respect to Martin measure. Since we will care only about the behavior of functions a.e., we will occasionally deal with functions which are only defined a.e.

For Turing invariant $f, g: 2^{\omega} \rightarrow 2^{\omega}$, let $f \leq_{m} g$ if and only if $f(x) \leq_{T} g(x)$ a.e. Similarly, $f \equiv_{m} g$ if and only if $f(x) \equiv_{T} g(x)$ a.e. Say that $f$ is increasing a.e. if $f(x) \geq_{T} x$ a.e. Finally, say that $f$ is constant a.e. if there exists a $y \in 2^{\omega}$ such that $f(x) \equiv_{T} y$ a.e. (i.e. the induced function on Turing degrees is constant a.e.).

We are now ready to state Martin's conjecture on Turing invariant functions.

Conjecture 1.1 (Martin [15, p. 281]). Assume ZF + DC + AD. Then

I. If $f: 2^{\omega} \rightarrow 2^{\omega}$ is Turing invariant, and $f$ is not increasing a.e. then $f$ is constant a.e.

II. $\leq_{m}$ prewellorders the set of Turing invariant functions which are increasing a.e. If $f$ has $\leq_{m}$-rank $\alpha$, then $f^{\prime}$ has $\leq_{m}$-rank $\alpha+1$, where $f^{\prime}(x)=f(x)^{\prime}$ for all $x$.

While Martin's conjecture remains open, significant progress has been made towards establishing its truth. Let $\varphi_{i}$ be the $i$ th partial recursive function. Say that $x \geq_{T} y$ via $i$ if $\varphi_{i}(x)=y$. Say that $x \equiv_{T} y$ via $(i, j)$ if $x \geq_{T} y$ via $i$ and $y \geq_{T} x$ via $j$. Suppose that $f$ is a Turing invariant function. Say that $f$ is uniformly Turing invariant if there exists a function $u: \omega^{2} \rightarrow \omega^{2}$ so that if $x \equiv_{T} y$ via $(i, j)$, then $f(x) \equiv_{T} f(y)$ via $u(i, j)$. Note that our definition of a uniformly Turing invariant function is slightly different that the definitions of some of the papers we reference 1 .

${ }^{1}$ In particular, the definition of uniformly Turing invariant that we give here is different than the definitions used in 25] and [26] (which also differ from each other). The definition in 25] requires only that there is a pointed perfect tree $T$ and a function $u: \omega^{2} \rightarrow \omega^{2}$ such that for all $x, y \in[T]$, if $x \equiv_{T} y$ via $(i, j)$, then $f(x) \equiv_{T} f(y)$ via $u(i, j)$. This definition is related to our definition in the following way:

Proposition. Let $f$ be Turing invariant. Then there exists a uniformly Turing invariant $g$ (using our definition) which is defined a.e. such that $g \equiv_{m} f$ if and only if there is a pointed perfect tree $T$ and a function $u: \omega^{2} \rightarrow \omega^{2}$ such that for all $x, y \in[T]$, if $x \equiv_{T} y$ via $(i, j)$, then $f(x) \equiv_{T} f(y)$ via $u(i, j)$.

Proof. For the forward direction, consider the function $\pi$ which maps $x$ to the lexicographically least $(i, j)$ such that $f(x) \equiv_{T} g(x)$ via $(i, j)$. Now use Lemma 3.5 to find a pointed perfect set on which $\pi$ is constant.

For the reverse direction, first let $[T]$ be the pointed perfect set on which $f$ is uniform. Pass to a uniformly pointed perfect tree $\hat{T} \subseteq T$ where there exists an $e$ such that for all $x \in[\hat{T}]$, we have $\varphi_{e}(x)=\hat{T}$. Now define the uniformly Turing invariant $g$ by composing $f$ with the canonical homeomorphism from $2^{\omega}$ to $[\hat{T}]$, and then restricting to the cone $\left\{x: x \geq_{T} \hat{T}\right\}$. 
The first progress on Martin's conjecture was made by Steel [26] and was continued by Slaman and Steel [25. They proved that Martin's conjecture is true when restricted to the class of uniformly Turing invariant functions.

Theorem 1.2 (Slaman and Steel [25]). Part I of Martin's conjecture holds for all uniformly Turing invariant functions.

Theorem 1.3 (Steel [26]). Part II of Martin's conjecture holds for all uniformly Turing invariant functions.

Theorems 1.2 and 1.3 also imply that Martin's conjecture is true when restricted to the larger class of functions $f$ so that $f \equiv_{m} g$ for some uniformly Turing invariant $g$. Steel has conjectured that this is true of all Turing invariant functions.

Conjecture 1.4 (Steel [26]). If $f: 2^{\omega} \rightarrow 2^{\omega}$ is Turing invariant, then there exists a uniformly Turing invariant $g$ which is defined a.e., and $f \equiv_{m} g$.

Assuming Conjecture 1.4. Steel [26] has computed the $\leq_{m}$-rank of many familiar such jump operators. Steel also proves that Conjecture 1.4 implies that if $f(x) \in$ $L[x]$ a.e., then $f$ has a natural normal form in terms of master codes in Jensen's J.

The original intent of Martin's conjecture was to be a precise way of stating that the only definable non-constant Turing invariant functions are the Turing jump and its transfinite iterates such as $x \mapsto x^{(\alpha)}$ for $\alpha<\omega_{1}, x \mapsto \mathcal{O}^{x}$, and $x \mapsto x^{\sharp}$. Becker has shown that Conjecture 1.4 precisely captures this idea. In [2], Becker defines the notion of a reasonable pointclass, and shows that for any such reasonable pointclass $\Gamma$, for every $x$, there is a universal $\Gamma(x)$ set, where $\Gamma(x)$ is the relativization of $\Gamma$ to $x$. (Such a universal set is not unique, but the universal $\Gamma(x)$ subset of $\omega$ will be unique up to Turing equivalence.) For instance, if we consider the pointclass of $\Pi_{1}^{1}$ sets, the universal $\Pi_{1}^{1}(x)$ subset of $\omega$ is $\mathcal{O}^{x}$. Becker has shown that the strictly increasing uniformly Turing invariant functions are precisely the functions which map $x$ to the universal $\Gamma(x)$ subset of $\omega$ for some reasonable pointclass $\Gamma$.

Theorem 1.5 (Becker [2]). Let $f: 2^{\omega} \rightarrow 2^{\omega}$ be a Turing invariant function so that $f(x)>_{T} x$ a.e. Then $f$ is uniformly Turing invariant if and only if there is a reasonable pointclass $\Gamma$, and a Turing invariant $g$ so that $g(x)$ is the universal $\Gamma(x)$ subset of $\omega$, and $f \equiv_{m} g$.

Suppose $f$ is Turing invariant. Then say that $f$ is order preserving if $x \geq_{T} y$ implies that $f(x) \geq_{T} f(y)$. Say that $f$ is uniformly order preserving if there exists a function $u: \omega \rightarrow \omega$ so that $x \geq_{T} y$ via i implies $f(x) \geq_{T} y$ via $u(i)$. It is clear that if $f$ is uniformly order preserving then $f$ is uniformly Turing invariant. A corollary of Becker's work is that for any Turing invariant $f$, there exists a uniformly Turing invariant $g$ so that $g \equiv_{m} f$ if and only if there exists a uniformly order preserving $h$ so that $h \equiv_{m} f$.

Two more cases of Martin's conjecture are known. They are especially interesting because they do not require uniformity assumptions.

Similar techniques along with Theorem 1.2 can be used to show that that for all Turing invariant $f$, there exists a $g \equiv_{m} f$ that is uniformly Turing invariant in our sense if and only if there exists an $h \equiv_{m} f$ that is uniformly Turing invariant in the sense of [26] (where $g$ and $h$ are both defined a.e.).

Thus, the differences between these definitions are harmless; we have not changed what it means for a function on Turing degrees to be represented a.e. by a uniformly invariant function. We use our definition because of its simplicity, and because it generalizes in Section 3.1 more readily than the definitions of [26] and 25]. 
Theorem 1.6 (Slaman and Steel [25]). If $f$ is a Borel order preserving Turing invariant function that is increasing a.e., then there exists an $\alpha<\omega_{1}$ so that $f(x) \equiv_{T} x^{(\alpha)}$ a.e.

The proof of this theorem uses a generalization of the Posner-Robinson theorem for iterates of the Turing jump up through $\omega_{1}$. To generalize this theorem beyond the Borel functions, it would be enough to generalize the Posner-Robinson theorem further through the hierarchy of jump operators. For instance, Woodin [33] has proved a generalization of the Posner-Robinson theorem for the hyperjump. This can be used to show that if $f$ is increasing and order preserving a.e., and not Borel, then $f(x) \geq_{T} \mathcal{O}^{x}$ a.e.

The last known case of Martin's conjecture is for all recursive functions.

Theorem 1.7 (Slaman and Steel 25]). Suppose $f(x) \leq_{T} x$ a.e. Then either $f(x) \equiv_{T} x$ a.e., or $f$ is constant a.e.

The proof of this theorem uses both game arguments and a significant amount of recursion theory. Generalizing this theorem past the $\boldsymbol{\Delta}_{1}^{0}$ functions appears to be difficult, and the proof does not give much of an idea of how to do this.

The special case of a degree invariant solution to Post's problem has also received considerable attention. Lachlan 16 has shown that there are no uniform solutions to Post's problem. This result predated Theorem 1.3. which generalized it. Downey and Shore [8] later put further restrictions on any possible solution. By using Theorem 1.3. they showed that any degree invariant solution to Post's problem must be low 2 or high ${ }_{2}$. On the positive side, Slaman and Steel (unpublished) have proved that there is a degree invariant solution to Post's problem restricted to the domain of $\Sigma_{3}^{0}$ sets. Finally, Lewis has constructed a degree invariant solution to Post's problem on a set of sufficiently generic degrees [17.

Martin's conjecture has also inspired a couple theorems for functions from $2^{\omega}$ to $P\left(2^{\omega}\right)$. Steel [26] has proved the existence of a natural prewellorder on inner model operators using the uniform case of Martin's conjecture. Slaman 24] has proved an analogue of Martin's conjecture for all Borel functions from $2^{\omega}$ to $P\left(2^{\omega}\right)$ satisfying certain natural closure conditions. This proof uses a technique that is reminiscent of Theorem 1.6, and relies on a sharpening of the generalized Posner-Robinson theorem due to Shore and Slaman [23].

The metamathematics of Martin's conjecture has been the source of some interesting results. Chong and $\mathrm{Yu}[5]$ have constructed uniformly Turing invariant $\Pi_{1}^{1}$ counterexamples to Martin's conjecture when the hypothesis of ZF $+\mathrm{DC}+\mathrm{AD}$ is replaced with $\mathrm{ZFC}+\mathrm{V}=\mathrm{L}$. They raised the question of the consistency strength of Theorem [1.3. Chong, Wang, and $\mathrm{Yu} 4$ have proved that the restriction of Theorem 1.3 to $\boldsymbol{\Pi}_{2 n+1}^{1}$ functions is equivalent to $\boldsymbol{\Sigma}_{2 n+2}^{1}$ determinacy for all $n \geq 0$.

Next, we will turn to applications of the above in the field of countable Borel equivalence relations. In this context, we will only need the restriction of Martin's conjecture to Borel functions. In what follows, when we assume Martin's conjecture, we will really mean that we assume its consequences for Borel functions. The following characterization of Borel Martin's conjecture is an easy consequence of Theorems 1.2 and 1.3 .

Theorem 1.8 (Slaman and Steel). The following are equivalent:

(1) Martin's conjecture restricted to Borel functions. 
(2) If $f$ is Borel and Turing invariant, then either $f$ is constant a.e., or there is an ordinal $\alpha<\omega_{1}$ so that $f(x) \equiv_{T} x^{(\alpha)}$ a.e.

(3) If $f$ is Borel and Turing invariant, then there exists a uniformly Turing invariant $g$ such that $f \equiv_{m} g$.

Assuming Martin's conjecture, there is a particular fact about Turing invariant functions that we will use several times. Given any subset $A$ of $2^{\omega}$, the $\equiv_{T^{-}}$ saturation of $A$ is defined to be the smallest Turing invariant set containing it. Let $f: 2^{\omega} \rightarrow 2^{\omega}$ be a countable-to-one function that is Turing-invariant. Then the $\equiv_{T}$-saturation of $\operatorname{ran}(f)$ must contain a Turing cone. This is because $f$ cannot be constant a.e. and so it must be that $f(x) \geq_{T} x$ a.e. Hence, the complement of the $\equiv_{T}$-saturation of $\operatorname{ran}(f)$ cannot contain a Turing cone.

1.2. Countable Borel equivalence relations. Turing equivalence is an example of a countable Borel equivalence relation. A Borel equivalence relation is an equivalence relation $E$ on a Polish space $X$ that is Borel as a subset of $X \times X$. A Borel equivalence relation is said to be countable if all its equivalence classes are countable.

Suppose $E$ and $F$ are Borel equivalence relations on the Polish spaces $X$ and $Y$ respectively, and $f: X \rightarrow Y$ is a function. $f$ is said to be a homomorphism from $E$ to $F$ if for all $x, y \in X$, we have $x E y \rightarrow f(x) F f(y)$. In this language, a Turing invariant function is a homomorphism from $\equiv_{T}$ to $\equiv_{T} . f$ is said to be a cohomomorphism from $E$ to $F$ if for all $x, y \in X$, we have $f(x) F f(y) \rightarrow x E y . f$ is said to be a reduction from $E$ to $F$ if $f$ is simultaneously a homomorphism and a cohomomorphism from $E$ to $F$. A reduction from $E$ to $F$ induces an injection from the quotient $X / E$ to the quotient $Y / F$. If the reduction $f$ is itself injective, then $f$ is said to be an embedding of $E$ into $F$.

$E$ is said to be Borel reducible to $F$, noted $E \leq_{B} F$, if there exists a Borel reduction from $E$ to $F$. The class of countable Borel equivalence relations under $\leq_{B}$ has a rich structure that has been studied extensively. See for instance the paper of Jackson, Kechris, and Louveau [11.

We will be particularly interested in the phenomenon of universality. A countable Borel equivalence relation $E$ is said to be universal if for all countable Borel equivalence relations $F$, we have $F \leq_{B} E$. It is known that there exist universal countable Borel equivalence relations $[6]$.

Martin's conjecture and the partial results surrounding it have turned out to have many connections with the field of countable Borel equivalence relations. Indeed, while Martin's conjecture says something very beautiful and fundamental about Turing reducibility and the hierarchy of definability, it is not so surprising that Martin's conjecture has been more applicable in this area, rather than in recursion theory. Martin's conjecture gives a complete classification of all homomorphisms from Turing equivalence to itself, and homomorphisms are a basic object of study in the area of countable Borel equivalence relations.

A countable Borel equivalence relation $E$ is said to be hyperfinite if $E=\bigcup_{i \in \omega} E_{i}$ where $E_{0} \subseteq E_{1} \subseteq \ldots$ is an increasing sequence of Borel equivalence relations with finite classes. Slaman and Steel 25] realized that if $\equiv_{T}$ was hyperfinite, this would allow one to construct counterexamples to Martin's conjecture. They showed that $\equiv_{T}$ is not hyperfinite and they proved that a Borel equivalence relation is hyperfinite if and only if it is induced by a Borel $\mathbb{Z}$ action. They obtained these results 
independently from the work that was beginning on the field of Borel equivalence relations at the time. Their last result is due independently to Weiss [32].

In [25, Slaman and Steel posed further structurability questions about Turing equivalence. These were answered by Kechris 12 via methods associated with the concept of amenability. Amenability has since played a large role in the study of Borel equivalence relations.

Kechris 13 has asked whether Turing equivalence is a universal countable Borel equivalence relation. An affirmative answer to this question would contradict Martin's conjecture, while Martin's conjecture implies that $\equiv_{T}$ is not universal. See [7 for a more thorough discussion of this topic. Essentially, if there is a reduction from $\equiv_{T} \sqcup \equiv_{T}$ to $\equiv_{T}$, then the range of the reduction on one of the copies of $\equiv_{T}$ must be disjoint from a cone.

A related question is due to Hjorth:

Question 1.9 (Hjorth [1, 11]). If $E$ and $F$ are countable Borel equivalence relations on the Polish space $X$, and $E$ is universal and $E \subseteq F$, then must $F$ be universal?

If this question has an affirmative answer, then $\equiv_{T}$ is universal; it is easy to find subsets of $\equiv_{T}$ that are universal. Of course, an affirmative answer to this question would have many more implications.

Let $E$ be a countable Borel equivalence relation on the Polish space $X$, and $\mu$ be a probability measure on $X$. Given a countable Borel equivalence relation $F$, we say that $E$ is $F$-ergodic with respect to $\mu$ if every Borel homomorphism from $E$ to $F$ maps a set of measure 1 into a single $F$-class. $E$ is said to be simply ergodic with respect to $\mu$ if it is $\Delta(Y)$-ergodic with respect to $\mu$ for every Polish space $Y$, where $\Delta(Y)$ is the equivalence relation of equality on $Y$. Define a subset $A$ of $X$ to be $E$-invariant if for all $x, y \in X$, if $x \in A$, and $x E y$, then $y \in A$. Equivalently, $E$ is ergodic with respect to $\mu$ if and only if every Borel $E$-invariant set has measure 0 or 1.

For the above definitions of ergodicity to make sense, $\mu$ can be a measure on merely the $\sigma$-algebra of $E$-invariant Borel subsets of $X$, as Martin measure is for $\equiv_{T}$. For example, $\equiv_{T}$ is ergodic with respect to Martin measure.

Strong ergodicity results for $\equiv_{T}$ and Martin measure would be very interesting. Let $E_{0}$ be the equivalence relation of equality mod finite on $2^{\omega}$. Thomas [30] has shown that if $\equiv_{T}$ is $E_{0}$-ergodic with respect to Martin measure, then $\equiv_{T}$ is not Borel bounded. Borel boundedness is closely connected to the long open increasing union problem for hyperfinite equivalence relations [3]. It is currently open whether there are any Borel equivalence relations that are not Borel bounded.

Thomas has shown that Martin's conjecture implies that $\equiv_{T}$ is $E_{0}$-ergodic with respect to Martin measure, and in fact, Martin's conjecture implies the strongest ergodicity for $\equiv_{T}$ that is possible with respect to Martin measure. If $E$ and $F$ are countable Borel equivalence relations, then a weak Borel reduction is a countable-toone Borel homomorphism from $E$ to $F$. If there exists a weak Borel reduction from $E$ to $F$, then we say that $E$ is weakly Borel reducible to $F$, and write $E \leq_{B}^{w} F$. A countable Borel equivalence relation $E$ is said to be weakly universal if $F \leq_{B}^{w} E$ for all countable Borel equivalence relations $F$. Clearly, if $E$ is weakly universal, then $\equiv_{T}$ is not $E$-ergodic with respect to Martin measure, since there is a countable-toone Borel homomorphism from $\equiv_{T}$ to $E$. Assuming Martin's conjecture, Thomas has proved the remarkable fact that the converse is true: 
Theorem 1.10 (Thomas [30]). Assume Martin's conjecture is true. Let E be any countable Borel equivalence relation. Then exactly one of the following hold:

(1) $E$ is weakly universal.

(2) $\equiv_{T}$ is E-ergodic, with respect to Martin measure.

The proof of this theorem uses the fact that Martin's conjecture implies that the saturation of the range of a countable-to-one Turing invariant function must contain a Turing cone. We will discuss Theorem 1.10 more in Section 3 ,

Martin's conjecture appears to be closely connected to the structure of the weakly universal countable Borel equivalence relations. Thomas [30] has shown that assuming Martin's conjecture, there are continuum many pairwise $\leq_{B}$-incomparable weakly universal countable Borel equivalence relations. These equivalence relations are products of the form $\equiv_{T} \times E_{\alpha}$ where $\left\{E_{\alpha}: \alpha \in 2^{\omega}\right\}$ is a family of non weakly universal countable Borel equivalence relations on $2^{\omega}$ so that if $\alpha \neq \beta$, then $E_{\alpha}$ is $E_{\beta}$-ergodic with respect to Lebesgue measure. The proof of this result uses Popa's cocycle superrigidity theorem to establish the existence of such a family of $E_{\alpha}$, and then applies Theorem 1.10 to prove the $\leq_{B}$-incomparability of the product equivalence relations.

Thomas has also used Martin's conjecture to investigate weak universality in another context. Say that a countable group $G$ is weakly action universal if there is a Borel action of $G$ on a Polish space $X$ so that the induced orbit equivalence relation $E_{G}^{X}$ is weakly universal. Assuming Martin's conjecture, Thomas has shown that $G$ is weakly action universal if and only if the conjugacy relation on the subgroups of $G$ is weakly universal 27 .

Aside from these results, the structure of the weakly universal countable Borel equivalence relations is poorly understood. For example, it is open whether there exists more than one weakly universal countable Borel equivalence relation up to Borel reducibility; this is equivalent to Hjorth's Question 1.9 having a negative answer.

An appeal of Martin's conjecture is that it provides a dimension of analysis that is completely orthogonal to measure theory. This is particularly interesting because all other known techniques for analyzing non-hyperfinite countable Borel equivalence relations are measure-theoretic. Assuming Martin's conjecture, Thomas [30] has proved that the complexity of a weakly universal countable Borel equivalence relation always concentrates on a nullset. This is strong evidence that techniques that are not purely measure-theoretic are needed to unravel the structure of the weakly universal countable Borel equivalence relations.

Theorem 1.11 (Thomas 30]). Assume Martin's conjecture. If $E$ is any weakly universal countable Borel equivalence relation on $X$, and $\mu$ is a Borel probability measure on $X$, then there is a Borel set $B \subseteq X$ with $\mu(B)=1$ so that $E \uparrow B$ is not weakly universal.

Thomas has applied this theorem to show that assuming Martin's conjecture, there does not exist a strongly universal countable Borel equivalence relation. We will discuss this result more in Section 3 ,

The partial results on Martin's conjecture have also found applications in the field of countable Borel equivalence relations. Thomas [29] has used Theorem 1.7 to show the nonexistence of continuous Borel reductions between several equivalence relations. These results are significant because in practice, most Borel reductions 
are continuous. Montalbán, Reimann, and Slaman (unpublished) have used Theorem 1.2 to show that $\equiv_{T}$ is not a uniformly universal countable Borel equivalence relations. We will discuss their theorem more in Section 3 .

\section{The UNIVERSALITY OF ARITHMETIC EQUIVALENCE}

It is natural to consider analogues of Martin's conjecture for other notions of degree. In particular, this makes sense for any degree notion that has a natural jump operator, and an analogue of Martin's measure (which generally exists by a proof similar to Martin's proof in [19]). For instance, we can replace Turing reducibility by arithmetic reducibility, the Turing jump with $x \mapsto x^{(\omega)}$, and Martin measure with the arithmetic cone measure.

In [25], Slaman and Steel note that their arguments for proving Theorems 1.2 and 1.3 adapt to the $\Delta_{n}^{1}$ degrees, and to the degrees of construtibility. However, they also note that their proofs do not work for arithmetic equivalence.

In later work, Slaman and Steel further investigated the analogue of Martin's conjecture for arithmetic equivalence. They showed that it is false, and the technique that they developed to build counterexamples adapted to prove a stronger result: that arithmetic equivalence is a universal countable Borel equivalence relation. It is this long unpublished result that we will give a proof of in this section. In Section 3, we will use this result to prove some theorems about universal countable Borel equivalence relations in general.

2.1. Basic definitions. The Cantor space, noted $2^{\omega}$, is the set of all functions from $\omega$ to 2 . There is a bijection between subsets of $\omega$ and elements of $2^{\omega}$; a subset of $\omega$ is represented by its characteristic function. In what follows, we freely move between these two representations. Say that $x \in 2^{\omega}$ has a limit if there exists an $i$ such that $\forall j>i, x(j)=x(i)$. Viewing $x$ has a subset of $\omega$, this is the same as saying that $x$ is finite or cofinite.

Given two reals $x, y \in 2^{\omega}$, the join of $x$ and $y$ is the real $x \oplus y$ defined by $(x \oplus y)(2 n)=x(n)$ and $(x \oplus y)(2 n+1)=y(n)$ for all $n \in \omega$. The join of finitely many reals is defined analogously. Fix a recursive bijection $\langle\cdot, \cdot\rangle$ from $\omega \times \omega \rightarrow \omega$. Let $x$ be a subset of $\omega$. The $n$th column of $x$, noted $x^{[n]}$, is the subset of $\omega$ defined by $x^{[n]}=\{m:\langle n, m\rangle \in x\}$. Conversely, if $\left\{x_{i}\right\}_{i \in \omega}$ are all reals, then the join of these reals, noted $\bigoplus_{i \in \omega} x_{i}$, is the real whose $n$th column is $x_{n}$.

Let $2^{<\omega}$ be the set of finite binary sequences. If $\sigma \in 2^{<\omega}$, then the length of $\sigma$, noted $|\sigma|$, is the domain of $\sigma$. If $x$ and $y$ are functions from $\omega$ to $2^{<\omega}$, define the join of $x$ and $y$ similarly to the above.

If $x, y \in 2^{\omega}$, then $x$ is said to be arithmetically reducible to $y$, noted $x \leq_{A} y$, if there is an $n$ so that $x$ has a $\Sigma_{n}^{0}$ definition relative to $y$. Equivalently, $y \geq_{A} x$ if there is an $n$ so that $y^{(n)} \geq_{T} x$, where $y^{(n)}$ is the $n$th iterate of the Turing jump relative to $y$. The associated countable Borel equivalence relation is called arithmetic equivalence and is noted $\equiv_{A}$.

2.2. The proof. Let $F_{2}$ be the free group on two generators. We define the countable Borel equivalence relation $E\left(F_{2}, 2\right)$ on $2^{F_{2}}$ in the following way: for all $x, y \in 2^{F_{2}}$, let $x E\left(F_{2}, 2\right) y$ if and only if there exists a $g \in F_{2}$ so that $x(h)=y(g h)$ for all $h \in F_{2}$. By a theorem of Dougherty, Jackson, and Kechris [6], this is a universal countable Borel equivalence relation. In order to show that arithmetic equivalence is universal, we shall construct a Borel embedding $f: 2^{F_{2}} \rightarrow 2^{\omega}$ of 
$E\left(F_{2}, 2\right)$ into $\equiv_{A}$. The particular properties of $E\left(F_{2}, 2\right)$ will be unimportant to the proof which would work equally well with any equivalence relation generated by the Borel action of a finitely generated group.

When constructing $f$, we must satisfy two conflicting requirements: we must make $f$ both a homomorphism and a cohomomorphism. In making $f$ a homomorphism, we must ensure that if $x E\left(F_{2}, 2\right) y$, then $f(x) \equiv_{A} f(y)$. Let $\left\{w_{i}\right\}_{i \in \omega}$ be a recursive listing of all the words in $F_{2}$. We will ensure that $f$ is a homomorphism by fixing a way of "coding" $f\left(w_{i} \cdot x\right)$ into $f(x)$, for every $i$.

An obvious method of coding would be as follows. Let $g: 2^{F_{2}} \rightarrow 2^{\omega}$ be any Borel function. Then define $\tilde{g}: 2^{F_{2}} \rightarrow 2^{\omega}$ to be

$$
\tilde{g}(x)=\bigoplus_{i \in \omega} g\left(w_{i} \cdot x\right)
$$

Given any $g$, we see that $\tilde{g}$ is a homomorphism from $E\left(F_{2}, 2\right)$ to $\equiv_{A}$; from $\tilde{g}(x)$, we can obtain any $\tilde{g}\left(w_{i} \cdot x\right)$ by recursively permuting columns. The task, then, would be to construct a Borel $g$ so that the associated $\tilde{g}$ was also a cohomomorphism.

Unfortunately, this approach is doomed to failure. If such a $\tilde{g}$ was a Borel reduction of $E\left(F_{2}, 2\right)$ to $\equiv_{A}$, it would also be a Borel reduction of $E\left(F_{2}, 2\right)$ to $\equiv_{T}$, and it would be a uniform reduction. Montalbán, Reimann, and Slaman have shown this is impossible. We will discuss their result more in Section 3 .

Essentially, the problem is that the above coding is too easy to unravel compared to how powerful arithmetic reductions are (or even how powerful Turing reductions are). The coding we use must evidently match the power of arithmetic equivalence more closely.

Our failed attempt above is interesting in the context of Hjorth's Question 1.9 . Suppose $E$ and $F$ are countable Borel equivalence relations, and $E \subseteq F$. A plausible intuition as to why the universality of $E$ would imply the universality of $F$ is as follows: it might be that any coding mechanism we could use to prove $E$ universal must also work to prove $F$ universal, simply by taking a more "generic" function that uses this coding. However, the above example shows that this is false; there are equivalence relations that are subsets of $\equiv_{A}$ for which the above coding mechanism can be used to prove universality.

The crux of the proof that $\equiv_{A}$ is universal is a method of coding so that for every $n$, there are only finitely many words $w_{i}$ so that $f\left(w_{i} \cdot x\right)$ is $\Sigma_{n}$ definable from $f(x)$. Hence, from the perspective of a $\Sigma_{n}$ reduction, $f(x)$ behaves as essentially a finite join. By taking a generic function $f$ for this type of coding, we can control these finite joins, and ensure that our $f$ is a cohomomorphism.

Definition 2.1. Given $y, z \in 2^{\omega}$, say that $z$ jump codes $y$ if for every $n, z^{[n]}$ has a limit, and $y(n)=\lim _{m} z^{[n]}(m)$. The Skolem function for this jump coding is the function from $\omega$ to $\omega$ that maps $n$ to the least $i$ such that $\forall j \geq i[z(\langle n, j\rangle)=z(\langle n, i\rangle)]$.

The name of this coding derives from the fact that if $z$ jump codes $y$, then $z^{\prime} \geq_{T}$ $y$. Indeed, using $z^{\prime}$ as an oracle, we can compute both $y$ and the Skolem function for this jump coding. Given $n$, find the least $i$ so that $\forall j>i[z(\langle n, j\rangle)=z(\langle n, i\rangle)]$, using the oracle $z^{\prime}$. Then the $n$th bit of $y$ is $z(\langle n, i\rangle)$.

Definition 2.2. Let $x: \omega \rightarrow 2^{<\omega}$ be any function. For any real $y \in 2^{\omega}$, define $J(x, y) \in 2^{\omega}$ to be the real that jump codes $y$ via $x$. Precisely, we mean that the 
$n$th column of $J(x, y)$ will be

$$
(J(x, y))^{[n]}= \begin{cases}x(n) 10000 \ldots & \text { if } \mathrm{y}(\mathrm{n})=0 \\ x(n) 01111 \ldots & \text { if } \mathrm{y}(\mathrm{n})=1\end{cases}
$$

Hence, $J(x, y)$ jump codes $y$, and the Skolem function for the jump coding is $n \mapsto|x(n)|+1$, where $|x(n)|$ is the length of the finite sequence $x(n)$.

If $p$ is a partial function from $\omega$ to $2^{<\omega}$ and $r$ is a partial function from $\omega$ to 2 with $\operatorname{dom}(p) \subseteq \operatorname{dom}(r)$, analogously define $J(p, r)$, a partial function from $\omega$ to 2 , where the $n$th column of $J(p, r)$ is undefined if $n \notin \operatorname{dom}(p)$.

The idea of coding a real as a limit of columns has a long history in recursion theory. The proof we will present uses jump codings of "depth" $\omega$. In this way, it is reminiscent of some constructions that have been used to investigate the structure $\left\langle\mathbf{D}, \leq_{T}, \prime\right\rangle$ of of the Turing degrees under $\leq_{T}$ and the jump operator. See the papers of Hinman and Slaman [10, and Montalbán [20.

In what follows, we will be using ideas from forcing in arithmetic, and in particular, reals with limited Cohen genericity. Let $\mathbb{P}_{\omega, 2<\omega}$ be the partial order of finite partial functions from $\omega$ to $2^{<\omega}$ ordered under inclusion. Say that a function $x$ from $\omega$ to $2^{<\omega}$ is arithmetically generic if it meets every arithmetically definable dense subset of $\mathbb{P}_{\omega, 2}<\omega$. Similarly, finitely many functions $x_{1}, \ldots x_{n}$ from $\omega$ to $2^{<\omega}$ are mutually arithmetically generic if $\left(x_{1}, \ldots, x_{n}\right)$ meets every arithmetically definable dense subset of $\left(\mathbb{P}_{\omega, 2<\omega}\right)^{n}$.

We begin with a lemma whose proof is standard for the subject:

Lemma 2.3. If $x, z$, and $w$ are mutually arithmetically generic functions from $\omega$ to $2^{<\omega}$, then for all $n \in \omega$ and $y \in 2^{\omega}$,

(1) $\left(0^{(n)} \oplus J(x, y) \oplus z\right)^{\prime} \equiv_{T} 0^{(n+1)} \oplus x \oplus y \oplus z$

(2) $0^{(n)} \oplus J(x, y) \oplus z \Varangle_{T} w$

Proof. We prove part 1. Let $\mathbb{P}_{\omega, 2}$ be the partial order of finite partial functions from $\omega$ to 2 ordered under inclusion.

Fix an $e$. Consider the set $D$ of pairs $(p, q) \in\left(\mathbb{P}_{\omega, 2<\omega}\right)^{2}$ such that for every $r \in \mathbb{P}_{\omega, 2}$ with $\operatorname{dom}(p)=\operatorname{dom}(r)$, either $\varphi_{e}\left(0^{(n)} \oplus J(p, r) \oplus q\right)$ halts, or for every extension of $(p, q, r)$ to $\left(p^{*}, q^{*}, r^{*}\right)$, we have that $\varphi_{e}\left(0^{(n)} \oplus J\left(p^{*}, r^{*}\right) \oplus q^{*}\right)$ does not halt. We claim that $D$ is is dense in $\left(\mathbb{P}_{\omega, 2<\omega}\right)^{2}$.

Suppose $(p, q) \in\left(\mathbb{P}_{\omega, 2<\omega}\right)^{2}$. We wish to show that that $(p, q)$ can be extended to meet $D$. Let $r_{1}, \ldots, r_{n}$ be a list of all elements of $\mathbb{P}_{\omega, 2}$ such that $\operatorname{dom}(p)=\operatorname{dom}\left(r_{i}\right)$. Let $s_{0}=\emptyset$, and $q_{0}=q$. We will define an increasing sequence $s_{1} \subseteq \ldots \subseteq s_{n}$ of elements of $\mathbb{P}_{\omega, 2}$ and an increasing sequence $q_{1} \subseteq \ldots \subseteq q_{n}$ of elements of $\mathbb{P}_{\omega, 2<\omega}$.

Inductively, for $1 \leq i \leq n$, consider $0^{(n)} \oplus J\left(p, r_{i}\right) \sqcup s_{i-1} \oplus q_{i-1}$, a partial function from $\omega$ to 2 . Either there no extension of this partial function that makes $\varphi_{e}$ halt relative to it, or there is a finite such extension. If there is such an extension, let it be $0^{(n)} \oplus J\left(p, r_{i}\right) \sqcup s_{i} \oplus q_{i}$, where $q_{i}$ extends $q_{i-1}$, where $s_{i}$ extends $s_{i-1}$, and the domain of $s_{i}$ is disjoint from $J\left(p, r_{i}\right)$. If there is no such extension, let $q_{i}=q_{i-1}$, and $s_{i}=s_{i-1}$.

Extend $p$ to any $\hat{p}$ so that for every $\langle j, k\rangle \in \operatorname{dom}\left(s_{n}\right)$, we have $\hat{p}(j)(k)=s_{n}(\langle j, k\rangle)$. Note that this means that for any $r \in \mathbb{P}_{\omega, 2}$ with $\operatorname{dom}(r)=\operatorname{dom}(\hat{p}), J(\hat{p}, r)$ will be an extension of $J(p, r) \sqcup s_{n}$. It is clear that $\left(\hat{p}, q_{n}\right)$ meets $D$.

If $x$ and $z$ are arithmetically generic, then for each $e, 0^{(n+1)} \oplus x \oplus y \oplus z$ can compute a place where $(x, z)$ meets $D$. Hence, from $0^{(n+1)} \oplus x \oplus y \oplus z$ we can compute the 
MARTIN'S CONJECTURE, $\equiv_{A}$, AND COUNTABLE BOREL EQUIVALENCE RELATIONS 11

$\Sigma_{1}^{0}$ theory of $0^{(n)} \oplus J(x, y) \oplus z$, and thus $0^{(n+1)} \oplus x \oplus y \oplus z \geq_{T}\left(0^{(n)} \oplus J(x, y) \oplus z\right)^{\prime}$. Obviously $\left(0^{(n)} \oplus J(x, y) \oplus z\right)^{\prime} \geq_{T} 0^{(n+1)} \oplus x \oplus y \oplus z$.

We now proceed to part 2, whose proof is similar to part 1. Fix an $e$. The dense set that $(x, z, w)$ must meet is the set of triples $(p, q, r) \in\left(\mathbb{P}_{\omega, 2}<\omega\right)^{3}$ such that for every $s \in \mathbb{P}_{\omega, 2}$ with $\operatorname{dom}(p)=\operatorname{dom}(s)$, there exists a $k$ such that $\varphi_{e}\left(0^{(n)} \oplus\right.$ $J(p, s) \oplus q)(k) \downarrow \neq r(k)$, or for every extension of $(p, q, s)$ to $\left(p^{*}, q^{*}, s^{*}\right)$, we have that $\varphi_{e}\left(0^{(n)} \oplus J(p, s) \oplus q\right)(k)$ does not halt. We leave the rest of the proof to the reader.

Note that for all $x_{0}, \ldots, x_{n}$ and $y_{0}, \ldots, y_{n}$, we have that

$$
J\left(x_{0}, y_{0}\right) \oplus \ldots \oplus J\left(x_{n}, y_{n}\right) \equiv_{T} J\left(x_{0} \oplus \ldots \oplus x_{n}, y_{0} \oplus \ldots \oplus y_{n}\right)
$$

and that if $x_{0}, \ldots, x_{n}$ and $z_{0}, \ldots, z_{n}$ are all mutually arithmetically generic, then $x_{0} \oplus \ldots \oplus x_{n}$ and $z_{0} \oplus \ldots \oplus x_{n}$ are mutually arithmetically generic. Therefore, we can conclude a more general fact:

Lemma 2.4. If $x_{0}, \ldots, x_{i}, z_{0}, \ldots, z_{j}$, and $w$ are mutually arithmetically generic functions from $\omega$ to $2^{<\omega}$, then for all $n \in \omega$ and $y_{0}, \ldots, y_{i} \in 2^{\omega}$

(1) $\left(0^{(n)} \oplus J\left(x_{0}, y_{0}\right) \oplus \ldots \oplus J\left(x_{i}, y_{i}\right) \oplus z_{0} \oplus \ldots \oplus z_{j}\right)^{\prime}$

$$
\equiv_{T} 0^{(n+1)} \oplus x_{0} \oplus \ldots \oplus x_{i} \oplus y_{0} \oplus \ldots \oplus y_{i} \oplus z_{0} \oplus \ldots z_{j} .
$$

(2) $0^{(n)} \oplus J\left(x_{0}, y_{0}\right) \oplus \ldots \oplus J\left(x_{i}, y_{i}\right) \oplus z_{0} \oplus \ldots \oplus z_{j} \Varangle_{T} w$

We are ready to prove the universality of arithmetic equivalence.

Theorem 2.5 (Slaman and Steel). $\equiv_{A}$ is a universal countable Borel equivalence relation.

Proof. Let $F_{2}=\langle a, b\rangle$. To prove this theorem, we will construct a Borel embedding of $E\left(F_{2}, 2\right)$ into $\equiv_{A}$. Let $g: 2^{F_{2}} \rightarrow\left(2^{<\omega}\right)^{\omega}$ be a Borel function so that for every distinct $x_{0}, \ldots, x_{n} \in 2^{F_{2}}$, we have that $g\left(x_{0}\right), \ldots, g\left(x_{n}\right)$ are all mutually arithmetically generic functions from $\omega$ to $2^{<\omega}$. The definition of the embedding $f: 2^{F_{2}} \rightarrow 2^{\omega}$ is as follows:

$$
f(x)=J\left(g(x), f(a \cdot x) \oplus f\left(a^{-1} \cdot x\right) \oplus f(b \cdot x) \oplus f\left(b^{-1} \cdot x\right)\right) .
$$

Note that while our definition of $f$ is self-referential, it is not circular, as one can see by repeatedly expanding the terms involving $f$ on the right hand side, using the definition of $f$.

First, $f$ is a homomorphism. Recall that for all $x$ and $y, J(x, y)^{\prime} \geq_{T} y$. Hence, if $x=w \cdot y$ where $w$ is a word of $F_{2}$ of length $n$, then $(f(x))^{(n)} \geq_{T} f(y)$.

Thus, we simply need to show that $f$ is a cohomomorphism. That is, if $x$ and $y$ are not $E\left(F_{2}, 2\right)$ equivalent, then $f(x)$ and $f(y)$ are not arithmetically equivalent. Let $\left\{w_{i}:\left|w_{i}\right|<n\right\}$ be all words in $F_{2}$ of length $<n$, and let $\left\{w_{i}:\left|w_{i}\right|=n\right\}$ be all words in $F_{2}$ of length $n$. Then

$$
(f(x))^{(n)} \equiv_{T} 0^{(n)} \oplus \bigoplus_{\left\{w_{i}:\left|w_{i}\right|<n\right\}} g\left(w_{i} \cdot x\right) \oplus \bigoplus_{\left\{w_{i}:\left|w_{i}\right|=n\right\}} f\left(w_{i} \cdot x\right)
$$

as one can see by inductively using part 1 of Lemma 2.4. (Recall that by definition, $f(z)$ is of the form $J(g(z), w)$ for some $w$, and $g(z)$ is part of our set of mutual generics). Hence, if $x$ and $y$ are not $E\left(F_{2}, 2\right)$ equivalent, by part 2 of Lemma 2.4 we see $(f(x))^{(n)} \Varangle_{T} g(y)$ for all $n$. Hence, $(f(x))^{(n-1)} \Varangle_{T} f(y)$, since $f(y)^{\prime} \geq_{T} g(y)$. 
The original proof of the existence of pathological arithmetically invariant functions was a similar construction to produce an embedding of $\equiv_{A}$ into itself. Note that the range of the embedding in Theorem 2.5 is disjoint from the arithmetic cone $\left\{x: x \geq_{A} 0^{\omega}\right\}$. Hence, embedding $\equiv_{A}$ into itself via this technique produces an injective arithmetically invariant function whose range is disjoint from an arithmetic cone.

\section{Corollaries And open PRoblems}

A measure analogous to Martin measure exists for arithmetic equivalence. It is called the arithmetic cone measure. An arithmetic cone is a set of the form $\left\{x: x \geq_{A} y\right\}$ for some $y$. An arithmetically invariant set has measure 1 with respect to the arithmetic cone measure if it contains an arithmetic cone, otherwise it has measure 0. Martin's proof in [19] still works when Turing reducibility is replaced by arithmetic reducibility. Hence, this function is indeed a measure on the $\sigma$-algebra of arithmetically invariant sets.

The proof that arithmetic equivalence is universal relativizes. That is, for every $x$, arithmetic equivalence relative to $x$ is universal. Equivalently, arithmetic equivalence restricted to any arithmetic cone is universal. Using this fact, we can obtain several interesting corollaries about universal countable Borel equivalence relations in general. The results in this section are due to the first author.

Jackson, Kechris, and Louveau [11] have asked the following question: suppose $E$ is a universal countable Borel equivalence relation on $X$, and $B$ is an $E$-invariant Borel subset of $X$. Is one of $E \uparrow B$ or $E \uparrow(X \backslash B)$ universal? The answer is yes, and we prove a stronger fact, originally posed as a question by Thomas [31, question $3.20]$.

Theorem 3.1. Suppose $X$ and $Y$ are Polish spaces, $E$ is a universal countable Borel equivalence relation on $X$, and $f: X \rightarrow Y$ is any Borel homomorphism from $E$ to $\Delta(Y)$, where $\Delta(Y)$ is the relation of equality on $Y$. Then there exists a $y \in Y$ so that the restriction of $E$ to $f^{-1}(y)$ is a universal countable Borel equivalence relation.

Proof. First, note that it is enough to prove this for arithmetic equivalence. Let $E$ and $f$ be as in the statement of the theorem, and let $g: 2^{\omega} \rightarrow X$ be a Borel reduction from $\equiv_{A}$ to $E$. If arithmetic equivalence restricted to $(f \circ g)^{-1}(y)$ is universal, then $E$ restricted to $f^{-1}(y)$ is universal.

Now let $f$ be a homomorphism from $\equiv_{A}$ to $\Delta(Y)$. Since arithmetic equivalence is ergodic with respect to the arithmetic cone measure, there must be a $y \in Y$ so that $f^{-1}(y)$ contains an arithmetic cone. Arithmetic equivalence restricted to this set is thus universal.

The use of Borel determinacy in our proof raises an interesting metamathematical question: must any proof of this theorem use Borel determinacy? For instance, one could ask whether Theorem 3.1 implies Borel determinacy over some simple base theory. We ask a weaker question of whether Theorem 3.1 shares a metamathematical property of Borel determinacy:

Question 3.2. Does a proof of Theorem 3.1 require the existence of $\omega_{1}$ iterates of the powerset of $\omega$ ? 
Let $E$ be a countable Borel equivalence relation, and suppose $f$ is a Borel homomorphism from $\equiv_{A}$ to $E$. Then $f$ is also a Borel homomorphism from $\equiv_{T}$ to

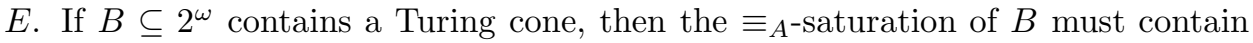
an arithmetic cone. It is therefore possible to use ergodicity results about Turing equivalence and Martin measure to obtain ergodicity results about arithmetic equivalence and the arithmetic cone measure. We will apply Thomas' Theorem 1.10 in this way to prove an analogous sort of ergodicity result for all universal countable Borel equivalence relations.

Theorem 3.3. Assume Martin's conjecture is true. Suppose E is a universal countable Borel equivalence relation, and $F$ is an arbitrary countable Borel equivalence relation. Then exactly one of the following holds:

(1) $F$ is weakly universal.

(2) For every Borel homomorphism $f$ of $E$ into $F$, there is a single F-class whose preimage $B$ has the property that $E \uparrow B$ is universal.

Proof. As in the proof of Theorem 3.1 we only need to prove this when $E$ is $\equiv_{A}$. Let $f$ be a homomorphism from $\equiv_{A}$ to $F$. Then $f$ is also a homomorphism from $\equiv_{T}$ to $F$, and hence by Theorem 1.10, either $F$ is weakly universal, or there is a single $F$-class whose preimage $B$ contains a Turing cone. In this latter case, since $f$ is also a homomorphism from $\equiv_{A}$ to $F$, the preimage of this single $F$-class contains the $\equiv_{A}$-saturation of this Turing cone which is an arithmetic cone. Hence, since $B$ contains an arithmetic cone, $\equiv_{A}\lceil B$ is universal.

In 30, Thomas proved a variant of this theorem where the assumption that $E$ is universal is changed to say $E$ is weakly universal, and option 2 is changed to say that $E \uparrow B$ is weakly universal. Theorem 3.3 strengthens this fact; by a result of Miller and Kechris [31, $\mathrm{E}$ is a weakly universal countable Borel equivalence relation if and only if there exists an $F \subseteq E$ that is a universal countable Borel equivalence relation.

In the proof of Theorem 3.3 , we have used the ergodicity of $\equiv_{T}$ that follows from Martin's conjecture. However, we only need the weaker ergodicity which Martin's conjecture implies for arithmetic equivalence. We isolate this in the following conjecture. It may be that it is easier to prove ergodicity results for arithmetic equivalence and the arithmetic cone measure than it is for Turing equivalence and Martin measure.

Conjecture 3.4. Let $E$ be any countable Borel equivalence relation. Then exactly one of the following holds:

(1) $E$ is weakly universal.

$(2) \equiv_{A}$ is E-ergodic, with respect to the arithmetic cone measure.

A special case of the above conjecture is quite interesting. Thomas [30] has raised the question of whether $\equiv_{T}$ is $E_{0}$-ergodic with respect to Martin measure. It is weaker to ask whether arithmetic equivalence is $E_{0}$-ergodic with respect to the arithmetic cone measure, but this would have similarly nice consequences. For example, it would imply that $\equiv_{A}$ is not Borel bounded, and also that option 2 in Theorem 3.3 holds when $F$ is $E_{0}$ without the assumption of Martin's conjecture.

For our next application, we will need to recall some facts about pointed perfect sets. Recall that a perfect subset of a Polish space $X$ is a closed subset of $X$ with no isolated points. Every perfect subset of $2^{\omega}$ can be realized as the paths $[T]$ through 
some infinite perfect tree $T$ in $2^{<\omega}$. A pointed perfect tree $T$ is a tree $T$ so that for all $x \in[T], x \geq_{T} T$. A pointed perfect set is the paths $[T]$ through some pointed perfect tree $T$. Pointed perfect sets arise naturally in determinacy arguments, and have many nice properties.

Given a perfect subset $[T]$ of $2^{\omega}$, it is clear that $2^{\omega}$ is homeomorphic to $[T]$ via a canonical homeomorphism that preserves the ordering on $2^{\omega}$. This homeomorphism will preserve the Turing degrees above $T$ if $T$ is pointed. That is, let $f: 2^{\omega} \rightarrow[T]$ be this canonical homeomorphism. If $T$ is a pointed perfect tree, then for all $x \geq_{T} T$, we have $x \equiv_{T} f(x)$, since both $x$ and $f(x)$ can compute a representation of $T$ and hence also a representation of $f$. The analogous facts also hold for arithmetic equivalence. In particular, if $T$ is a pointed perfect tree, and $f: 2^{\omega} \rightarrow[T]$ is the canonical homeomorphism from $2^{\omega}$ to $[T]$, then for all $x \geq_{A} T$, we will have $x \equiv_{A} f(x)$. Hence, the restriction of $\equiv_{A}$ to any pointed perfect set is still a universal countable Borel equivalence relation.

We will use the following lemma, which illustrates a useful feature of pointed perfect sets in determinacy arguments. The proof of this lemma is a slight variation of Martin's cone theorem in [19].

Lemma 3.5 (Martin [19]). Assume $\mathrm{ZF}+\mathrm{DC}+\mathrm{AD}$. Then given any function $\pi: 2^{\omega} \rightarrow \omega$, there exists a pointed perfect set on which $\pi$ is constant.

Proof. Consider the game where I plays $e \in \omega$ followed by $x \in 2^{\omega}$, and II plays $y \in 2^{\omega}$, where the players alternate playing bits of these reals as usual. Let II lose unless $y \geq_{T} x$, and if the game is not decided by this condition, then I wins if and only if $x \geq_{T} y$, and $\pi(x)=e$.

Given any strategy $\tau$ for II, I can win by playing $\pi(\tau)$ followed by $\tau$. Hence, I wins this game. Let $\sigma$ be a winning strategy for I. Then our pointed perfect set is the set of I's winning plays against II playing $\left\{y: y \geq_{T} \sigma\right\}$.

If $E$ and $F$ are countable Borel equivalence relations, then we say that $E$ is Borel embeddable in $F$ and write $E \sqsubseteq_{B} F$ if there exists a Borel embedding of $E$ into $F$. We can use the above lemma to derive the following fact about universality for embeddings.

Theorem 3.6. Let $E$ be a universal countable Borel equivalence relation. Then given any countable Borel equivalence relation $F$, it must be that $F \sqsubseteq_{B} E$. That is, not only is $F \leq_{B} E$ (since $E$ is universal), we can always find an injective Borel reduction.

Proof. First recall that the reduction from $E\left(F_{2}, 2\right)$ to $\equiv_{A}$ in the proof of Theorem 2.5 is actually a Borel embedding, and not merely a Borel reduction. Of course, this remains true when the proof is relativized to any pointed perfect set. Recall also that Dougherty, Jackson, and Kechris [6] have shown that every countable Borel equivalence relation embeds into $E\left(F_{2}, 2\right)$. Hence, it will be enough to show that there is an embedding of $\equiv_{A}$ restricted to some pointed perfect set into $E$.

Since $E$ is countable universal, there is a Borel reduction $f$ from $\equiv_{A}$ to $E$. Using Lusin-Novikov uniformization $\left(18.10,18.15\right.$ in [14]), split $2^{\omega}$ into countably many Borel pieces $\left\{B_{i}\right\}_{i \in \omega}$ so that $f$ is injective on each $B_{i}$. One of these $B_{i}$ must contain a pointed perfect set by Lemma 3.5 .

This theorem is an interesting counterpoint to the following theorem of Thomas: 
Theorem 3.7 (Thomas [28]). There exist countable Borel equivalence relations $E$ and $F$ such that the equivalence classes of both $E$ and $F$ are all infinite, and $E \leq_{B} F$, and $F \leq_{B} E$, but it is not the case that $E \sqsubseteq_{B} F$.

Lemma 3.5 also gives an easy proof of the following:

Theorem 3.8. Suppose $E$ is a universal countable Borel equivalence relation on a Polish space $X$, and let $\left\{B_{i}\right\}_{i \in \omega}$ be a partition of $X$ into countably many (not necessarily E-invariant) Borel pieces. Then there exists some $i$ such that $E \uparrow B_{i}$ is a universal countable Borel equivalence relation.

Proof. As in Theorem 3.1. we only need to prove this for $\equiv_{A}$. Let $\left\{B_{i}\right\}_{i \in \omega}$ be a partition of $2^{\omega}$ into countably many Borel pieces. By Lemma 3.5 above, one of these pieces must contain a pointed perfect set, and the restriction of $\equiv_{A}$ to any pointed perfect set is countable universal.

If the $B_{i}$ in the above theorem are all $E$-invariant, this theorem follows from Theorem 3.1. From this, we could also conclude the general case since for any Borel $B, E \uparrow B$ is universal if and only if $E \uparrow[B]_{E}$ is universal, where $[B]_{E}$ is the $E$-saturation of $B$.

Theorem 3.8 associates two natural $\sigma$-ideals to every countable Borel equivalence relation.

Definition 3.9. Let $E$ be a countable Borel equivalence relation on the Polish space $X$. Define the non-universal ideal of $E$ to be the Borel subsets $B$ of $X$ on which $E \uparrow B$ is not universal. Define the non-weakly-universal ideal of $E$ to be the Borel subsets $B$ of $X$ on which $E \uparrow B$ is not weakly universal.

We will discuss these $\sigma$-ideals more in what follows. They seem to be important for developing the theory of universal and weakly universal countable Borel equivalence relations.

Let $E$ be a countable Borel equivalence relation on the Polish space $X$ which is equipped with an invariant ergodic Borel probability measure $\mu$. Say that $E$ is strongly universal if $E \uparrow B$ is universal for every Borel $B \subseteq X$ with $\mu(B)=1$. In [31, Thomas asked whether there exists a strongly universal countable Borel equivalence relation. Thomas later settled this question under the assumption of Martin's conjecture using Theorem 1.11. We are able to prove a weaker theorem without the assumption of Martin's conjecture.

Theorem 3.10. Let $E$ be a universal countable Borel equivalence relation on the space $X$, and let $\mu$ be a Borel probability measure on $X$. Then there is a measure 0 subset $B$ of $X$ for which $E \uparrow B$ is a universal countable Borel equivalence relation.

First, recall the following theorem of Sacks (we give the relativized version of the theorem):

Theorem 3.11 (Sacks 21]). If $\mu$ is a Borel probability measure on $2^{\omega}$, then for all $x \in 2^{\omega}$ such that there exists a representation $y \in 2^{\omega}$ of $\mu$ such that $x>_{T} y$, the cone $\left\{z: z \geq_{T} x\right\}$ has $\mu$-measure 0 .

Hence, for any Borel probability measure, sufficiently complicated cones are always nullsets. The same theorem is also true when Turing reducibility is replaced with arithmetic reducibility. One way to see this is to first replace our measure $\mu$ with an $\equiv_{A}$-quasi-invariant measure $\nu$ that dominates $\mu$. Then we can find a Turing 
cone with $\nu$ measure 0 , and the $\equiv_{A}$-saturation of this cone will be the arithmetic cone with the same base.

Sacks' theorem implies that Martin measure and likewise the arithmetic cone measure cannot be extended to probability measures on all the Borel sets of $2^{\omega}$. Pointed perfect sets seem to be as close as we can get to being able to measure arbitrary Borel sets using these measures.

Proof of Theorem 3.10: Again, we need only prove this for arithmetic equivalence; given any other universal countable Borel equivalence relation $E$ on the space $X$ with Borel probability measure $\mu$, by Theorem 3.6 let $f$ be a Borel embedding from $\equiv_{A}$ to $E$. Presuming the range of $f$ has positive $\mu$-measure, let $\nu$ be the measure on $2^{\omega}$ defined by $\nu(A)=\frac{1}{\mu\left(f\left(2^{\omega}\right)\right)} \mu(f(A))$. If $\equiv_{A}\lceil B$ is universal and $\nu(B)=0$, then $E\lceil f(B)$ is also universal, and $\mu(f(B))=0$.

As we have shown above, given any Borel probability measure $\mu$ on $\equiv_{A}$, there is an arithmetic cone with measure 0 , and $\equiv_{A}$ restricted to this cone is universal.

The extra leverage that Thomas gets by assuming Martin's conjecture is that for all Borel $B, \equiv_{T} \uparrow B$ is weakly universal if and only if $B$ contains a pointed perfect set. Hence if $B$ is $\equiv_{T}$-invariant, then $\equiv_{T} \uparrow B$ is weakly universal if and only if $\equiv_{T} \uparrow\left(2^{\omega} \backslash B\right)$ is not weakly universal.

This exact classification of the non-weakly-universal ideal for $\equiv_{T}$ that follows from Martin's conjecture seems very useful.

Question 3.12. Are there "nice" characterizations of the non-weakly-universal ideals of naturally occurring weakly universal countable Borel equivalence relations?

One could also ask the same question for universal countable Borel equivalence relations, and the non-universal ideal. Theorem [3.10 seems to rule out characterizations that are based purely on measure theory. The fact that these ideals do not seem to be measure-theoretic is very interesting, since all known theorems in the field of countable Borel equivalence relations that distinguish between nonhyperfinite countable Borel equivalence relations are based on measure theory.

Marks 18 raises a question that seems to be relevant. Define a countable Borel equivalence relation $E$ to be measure universal if for every countable Borel equivalence relation $F$ on a Polish space $X$ equipped with a Borel probability measure $\mu$, there exists a $B \subseteq X$ that is $F$-invariant, and $\mu(B)=1$, so that $F\lceil B$ is Borel reducible to $E$.

Question 3.13 (Marks [18]). If $F$ is a countable Borel equivalence relation that is measure universal, is $F$ universal?

This question was motivated by a result in Marks 18 that many-one equivalence and recursive isomorphism are measure universal. It remains open whether these equivalence relations are universal.

3.1. Some questions on uniformity. Borel Martin's conjecture reduces purely to a question about uniformity, as shown in Theorem 1.8. If we embrace Martin's conjecture and ponder what larger principle it might embody, we are naturally led to the possibility that similar principles of uniformity might exist amongst a much wider class of equivalence relations, even though the original form of Martin's conjecture appears to hinge on specific properties of Turing equivalence that do not generalize to many equivalence relations. This is an intriguing possibility that 
would lead to a compelling theory providing a systematic way to explain many phenomena. In this section, we shall adopt such a viewpoint and pose several questions about uniformity in broader contexts. Our questions will be phrased so that affirmative answers would be the most natural from the above perspective. However, even negative answers would be very interesting as they might provide starting points for constructing counterexamples to Martin's conjecture.

Admittedly, we currently have little evidence supporting the viewpoint we shall outline. Such questions of uniformity are presently poorly understood, and these issues appear quite deep. We know of no general theorems in this area, and not even any theorems in specific cases, beyond the work in 25. Likewise, there are few examples of nonuniformity which seem to have much bearing on the questions we will ask.

Consider, for instance, the case of arithmetic equivalence. We have seen above that the analogue of Martin's conjecture fails for arithmetic equivalence. However, many questions about arithmetically invariant functions remain, and chief among them is the arithmetic analogue of Conjecture 1.4. Say that an arithmetically invariant function $f$ is uniformly arithmetically invariant if there exists a function $u: \omega^{2} \rightarrow \omega^{2}$ such that if $x \equiv_{A} y$ via $(i, j)$, then $f(x) \equiv_{A} f(y)$ via $u(i, j)$.

Question 3.14 ( $\mathrm{ZF}+\mathrm{DC}+\mathrm{AD})$. If $f: 2^{\omega} \rightarrow 2^{\omega}$ is arithmetically invariant, then is there a uniformly arithmetically invariant $g$ which is defined on an arithmetic cone so that $f(x) \equiv_{A} g(x)$ on an arithmetic cone?

Little is known about this question. The pathological arithmetically invariant functions that were constructed in Section 2.2 are all uniformly invariant, and the technique used to construct them gives no hint about questions of nonuniformity. Likewise, the following conjecture is particularly intesting in light of the earlier results in this section:

Conjecture 3.15. If $f$ is a Borel homomorphism from $\equiv_{A}$ to $\equiv_{T}$, then there exists a Borel homomorphism $g$ from $\equiv_{A}$ to $\equiv_{T}$ so that $f(x) \equiv_{A} g(x)$ on an arithmetic cone and $g$ is uniform in the sense that there exists a function $u: \omega^{2} \rightarrow \omega^{2}$ so that for all $x$ and $y$, if $x \equiv_{A} y$ via $(i, j)$, then $g(x) \equiv_{T} g(y)$ via $u(i, j)$.

This conjecture is weaker than Borel Martin's conjecture, but it has many of the same consequences for the theory of Borel equivalence relations. For instance, it implies that Conjecture 3.4 is true, and hence that Theorem 3.3 is true without the assumption of Martin's conjecture. It also implies that Turing equivalence is not a universal countable Borel equivalence relation. This is because it rules out an embedding of $\equiv_{A}$ into $\equiv_{T}$; for all $\alpha$ with $\omega \leq \alpha<\omega_{1}$, the map $x \mapsto x^{(\alpha)}$ is not a reduction of arithmetic equivalence restricted to any arithmetic cone into $\equiv_{T}$.

It seems interesting to consider questions of uniformity analogous to Conjecture 3.15 for most other equivalence relations from recursion theory. Even more generally, we shall formulate such questions for arbitrary weakly universal countable Borel equivalence relations.

In the field of countable Borel equivalence relations, uniformity is usually discussed in the context where an equivalence relation $E$ is equipped with a group $G$ and a Borel action of $G$ that generates $E$. If $G$ is a countable group that acts on a Polish space $X$, define the orbit equivalence relation $E_{G}^{X}$ where $x E_{G}^{X} y$ if and only if there exists a $g \in G$ such that $g \cdot x=y$. By a result of Feldman and Moore [9], every countable Borel equivalence relation $E$ is of this form. 
In recursion theory, however, most equivalence relations (for instance $\equiv_{T}$ and $\equiv_{A}$ ) are not naturally generated by group actions. Further, while the FeldmanMoore theorem guarantees that it is possible to construct group actions that generate recursion-theoretic equivalence relations, it is often difficult to work with them using tools from recursion theory. Thus, when working with weakly universal equivalence relations, it seems natural to expand our definitions, since recursion-theoric equivalence relations seem so important in this context.

Definition 3.16. A generating family of partial Borel functions on a Polish space $X$ is a countable set $\left\{\phi_{i}\right\}$ of partial Borel functions on $X$ that is indexed by natural numbers (though we will omit the indexing for clarity), contains the identity function, and is closed under composition. Precisely, by partial Borel function on $X$, we mean that the domain of each $\phi_{i}$ is a Borel subset of $X$, and that the function $\phi_{i}: \operatorname{dom}\left(\phi_{i}\right) \rightarrow X$ is a Borel function. Such a generating family induces an equivalence relation $E_{\left\{\phi_{i}\right\}}^{X}$, where $x E_{\left\{\phi_{i}\right\}}^{X} y$ if and only if there exists $\phi_{i}$ and $\phi_{j}$ so that $\phi_{i}(x)=y$, and $\phi_{j}(y)=x$. In this case, say that $x E_{\left\{\phi_{i}\right\}}^{X} y$ via $(i, j)$.

We will use the notation $E_{\left\{\phi_{i}\right\}}^{X}$ to indicate a countable Borel equivalence relation on the Polish space $X$ that is induced by the generating family of partial Borel functions $\left\{\phi_{i}\right\}$.

For example, the Turing reductions are a generating family of partial Borel functions for $\equiv_{T}$, and the arithmetic reductions are a generating family of partial Borel functions for $\equiv_{A}$.

Definition 3.17. Suppose $E_{\left\{\phi_{i}\right\}}^{X}$ and $E_{\left\{\psi_{i}\right\}}^{Y}$ are countable Borel equivalence relations on the Polish spaces $X$ and $Y$ induced by the generating families of partial Borel functions $\left\{\phi_{i}\right\}$ and $\left\{\psi_{i}\right\}$. Say that a partial homomorphism $f: X \rightarrow Y$ is uniform (with respect to $\left\{\phi_{i}\right\}$ and $\left\{\psi_{i}\right\}$ ) if there exists a function $u: \omega^{2} \rightarrow \omega^{2}$ such that for all $x, y \in X$, if $x E_{\left\{\phi_{i}\right\}}^{X} y$ via $(i, j)$, then $f(x) E_{\left\{\psi_{i}\right\}}^{Y} f(y)$ via $u(i, j)$.

Let $E_{G}^{X}$ be a countable Borel equivalence relation generated by a Borel action of the countable group $G$. To each $g \in G$ we associate the Borel automorphism $\phi_{g}(x)=g \cdot x$ of $\mathrm{X}$, and so $E_{G}^{X}$ is the same as the equivalence relation $E_{\left\{\phi_{g}\right\}}^{X}$ given by the generating family $\left\{\phi_{g}\right\}$. Say that $E_{G}^{X}$ is freely generated if for all $g \in G$ and for all $x \in X$, if $g \cdot x=x$ then $g=1$. In the context where $E_{G}^{X}$ and $E_{H}^{Y}$ are freely generated countable Borel equivalence relations, then in the measure context, the uniformity defined above is equivalent to saying that the cocycle induced by a homomorphism between equivalence relations is cohomologous to a group homomorphism. Thus, the sort of uniformity implied by Martin's conjecture (and more general questions which we will soon discuss) shares something of the same spirit as cocycle supperrigidity which was introduced by Zimmer 34. Cocycle superrigidity has since played an important part in developing the theory of countable Borel equivalence relations.

We will now formulate an analogue of Conjecture 3.15 for homomorphisms between two arbitrary weakly universal countable Borel equivalence relations. In posing such a question, we need an equivalent of a set of Martin measure 1; a "large" subset of the underlying space on which we demand that uniformity is witnessed. Here, the natural candidate is an invariant Borel set such that the restriction of the equivalence relation to this set is still weakly universal. This notion agrees with 
Martin measure in the case of $\equiv_{T}$. The fact that the non-weakly-universal Borel sets form a $\sigma$-ideal is further evidence that this is a good notion of "largeness".

Note that it is certainly not the case that for every homomorphism $f$ between equivalence relations $E_{\left\{\phi_{i}\right\}}^{X}$ and $E_{\left\{\psi_{i}\right\}}^{Y}$, there exists a uniform homomorphism $g$ from $E_{\left\{\phi_{i}\right\}}^{X}$ to $E_{\left\{\psi_{i}\right\}}^{Y}$ so that $f(x) E_{\left\{\psi_{i}\right\}}^{Y} g(x)$ for all $x$. There are trivial counterexamples exploiting partiality of generating families. Even in the case of equivalence relations freely generated by the actions of countable groups, not every cocycle associated to a homomorphism of such equivalence relations is cohomologous to a group homomorphism.

We proceed to our question:

Question 3.18. Let $E_{\left\{\phi_{i}\right\}}^{X}$ and $E_{\left\{\psi_{i}\right\}}^{Y}$ be weakly universal countable Borel equivalence relations. Now suppose $f$ is a homomorphism from $E_{\left\{\phi_{i}\right\}}^{X}$ to $E_{\left\{\psi_{i}\right\}}^{Y}$. Must there be an $E_{\left\{\phi_{i}\right\}}^{X}$-invariant subset $B$ of $X$ such that $E_{\left\{\phi_{i}\right\}}^{X}\lceil B$ is weakly universal, and a uniform partial homomorphism $g: X \uparrow B \rightarrow Y$ from $E_{\left\{\phi_{i}\right\}}^{X}$ to $E_{\left\{\psi_{i}\right\}}^{Y}$ such that $f(x) E_{\left\{\psi_{i}\right\}}^{Y} g(x)$ for all $x \in B$ ?

As stated, an affirmative answer to this question does not seem to generalize Borel Martin's conjecture. For this to be the case, we also need a characterization of the non-weakly-universal ideal of Turing equivalence.

Definition 3.19. Suppose that $E_{\left\{\phi_{i}\right\}}^{X}$ is a weakly universal countable Borel equivalence relation. Say that $E_{\left\{\phi_{i}\right\}}^{X}$ is uniformly weakly universal if for every $E_{\left\{\psi_{i}\right\}}^{Y}$, there is a uniform weak Borel reduction of $E_{\left\{\psi_{i}\right\}}^{Y}$ to $E_{\left\{\phi_{i}\right\}}^{X}$.

A related definition will also be of interest to us:

Definition 3.20. Suppose that $E_{\left\{\phi_{i}\right\}}^{X}$ is a universal countable Borel equivalence relation. Say that $E_{\left\{\phi_{i}\right\}}^{X}$ is uniformly universal if for every $E_{\left\{\psi_{i}\right\}}^{Y}$, there is a uniform Borel reduction from $E_{\left\{\psi_{i}\right\}}^{Y}$ to $E_{\left\{\phi_{i}\right\}}^{X}$.

All known universal and weakly universal countable Borel equivalence relations are uniformly universal and uniformly weakly universal when equipped with natural generating families. For instance, $E\left(F_{2}, 2\right)$ is uniformly universal with respect to the shift action of $F_{2}$, arithmetic equivalence is uniformly universal with respect to the family of arithmetic reductions, and Turing equivalence is uniformly weakly universal with respect to the family of Turing reductions. Note that uniform universality and uniform weak universality both depend on the generating family that we use. In particular, every universal/weakly universal countable Borel equivalence relation $E$ is uniformly universal/uniformly weakly universal for some generating family, as one can see by applying Theorem 3.6.

Question 3.21. Suppose that $E_{\left\{\phi_{i}\right\}}^{X}$ is a weakly universal countable Borel equivalence relation. Must $E_{\left\{\phi_{i}\right\}}^{X}$ be weakly uniformly universal?

A positive answer to Question 3.21 would provide the characterization of the non-weakly-universal ideal of $\equiv_{T}$ that is needed so that a positive answer to Question 3.18 implies Borel Martin's conjecture.

Proposition 3.22. An affirmative answer to both Question 3.21 and Question 3.18 implies Borel Martin's conjecture. 
Proof. In what follows, equip $\equiv_{T}$ with the generating family of Turing reductions. Consider a $\equiv_{T}$-invariant subset $B$ of $2^{\omega}$ so that $\equiv_{T}\lceil B$ is weakly universal. Supposing Question 3.21 has a positive answer, there must be a uniform countable-to-one homomorphism from $\equiv_{T}$ to $\equiv_{T} \uparrow B$. Hence, $B$ must contain a cone.

Now let $f: 2^{\omega} \rightarrow 2^{\omega}$ be a homomorphism from $\equiv_{T}$ to $\equiv_{T}$. Supposing Question 3.18 has a positive answer, there is a weakly universal $\equiv_{T}$-invariant subset $B$ of $\equiv_{T}$ and a uniform homomorphism $g$ from $\equiv_{T}$ to $\equiv_{T}$ so that $f(x) \equiv_{T} g(x)$ on $B$. By the above, $B$ contains a Turing cone.

Versions of Questions 3.18 and 3.21 that extend beyond the Borel realm also seem interesting. For instance, one can consider these questions in the context of $\mathrm{ZF}+\mathrm{DC}+\mathrm{AD}^{+}$, where we use $\mathrm{AD}^{+}$as opposed to $\mathrm{AD}$ so that there is enough uniformization so that weakly universal countable equivalence relations exist. Here, positive answers to these questions would imply the analogue of Martin's conjecture for the $\Delta_{n}^{1}$ degrees and the degrees of construtibility via versions of Proposition 3.22 and Theorems 1.2 and 1.3 for these degree notions.

We finish by stating a question about uniform universality which is related to Question 3.21, and has arisen naturally in other work.

If we generate $\equiv_{T}$ by the family of Turing reductions, then by applying the uniform case of Martin's conjecture in Theorem 1.2, we see that there cannot be any uniform embedding of $\equiv_{T} \sqcup \equiv_{T}$ into $\equiv_{T}$. Hence, $\equiv_{T}$ is not uniformly universal for the generating family of Turing reductions. Montalbán, Reimann, and Slaman (unpublished) have generalized this fact to show that $\equiv_{T}$ is not uniformly universal when it is equipped with a certain generating group. The central technical obstacle in their work is adapting the game proofs of [25] to work with this group. Montalbán, Reimann, and Slaman posed the question of what role uniformity plays in universality proofs, and more broadly in the theory of countable Borel equivalence relations. Their question has been inspiration for much of this section. In this spirit, we ask the following:

Question 3.23. Suppose that $E_{\left\{\phi_{i}\right\}}^{X}$ is a universal countable Borel equivalence relation. Must $E_{\left\{\phi_{i}\right\}}^{X}$ be uniformly universal?

Uniform universality in this more general setting has also shown up naturally in recent work of Marks [18, who has shown that many-one equivalence is uniformly universal with respect to the generating family of many-one reductions if and only if a question in Borel combinatorics has an affirmative answer. A resolution of Question 3.23 would help clarify this situation.

\section{REFERENCES}

[1] Alessandro Andretta, Riccardo Camerlo, and Greg Hjorth, Conjugacy equivalence relation on subgroups, Fund. Math. 167 (2001), no. 3, 189-212. MR1815088 (2001m:03091)

[2] Howard Becker, A characterization of jump operators, J. Symbolic Logic 53 (1988), no. 3, 708-728. MR960994 (90a:03067)

[3] Charles M. Boykin and Steve Jackson, Borel boundedness and the lattice rounding property, Advances in logic, 2007, pp. 113-126. MR2322367 (2008e:03081)

[4] C. T. Chong, Wei Wang, and Liang Yu, The strength of the projective Martin conjecture, Fund. Math. 207 (2010), no. 1, 21-27. MR2576277 (2011g:03100)

[5] C. T. Chong and Liang $\mathrm{Yu}, A \Pi_{1}^{1}$-uniformization principle for reals, Trans. Amer. Math. Soc. 361 (2009), no. 8, 4233-4245. MR2500887 (2010b:03046)

[6] R. Dougherty, S. Jackson, and A. S. Kechris, The structure of hyperfinite Borel equivalence relations, Trans. Amer. Math. Soc. 341 (1994), no. 1, 193-225. MR1149121 (94c:03066) 
MARTIN'S CONJECTURE, $\equiv_{A}$, AND COUNTABLE BOREL EQUIVALENCE RELATIONS 21

[7] Randall Dougherty and Alexander S. Kechris, How many Turing degrees are there?, Computability theory and its applications (Boulder, CO, 1999), 2000, pp. 83-94. MR1770736 (2001d:03104)

[8] Rodney G. Downey and Richard A. Shore, There is no degree invariant half-jump, Proc. Amer. Math. Soc. 125 (1997), no. 10, 3033-3037. MR1401736 (97m:03073)

[9] Jacob Feldman and Calvin C. Moore, Ergodic equivalence relations, cohomology, and von Neumann algebras. I, Trans. Amer. Math. Soc. 234 (1977), no. 2, 289-324. MR0578656 (58 \#28261a)

[10] Peter G. Hinman and Theodore A. Slaman, Jump embeddings in the Turing degrees, J. Symbolic Logic 56 (1991), no. 2, 563-591. MR1133085 (93b:03072)

[11] S. Jackson, A. S. Kechris, and A. Louveau, Countable Borel equivalence relations, J. Math. Log. 2 (2002), no. 1, 1-80. MR1900547 (2003f:03066)

[12] Alexander S. Kechris, Amenable equivalence relations and Turing degrees, J. Symbolic Logic 56 (1991), no. 1, 182-194. MR1131739 (93c:03061)

[13] _ , The structure of Borel equivalence relations in Polish spaces, Set theory of the continuum (Berkeley, CA, 1989), 1992, pp. 89-102. MR1233813 (94h:03093)

[14] Classical descriptive set theory, Graduate Texts in Mathematics, vol. 156, SpringerVerlag, New York, 1995. MR1321597 (96e:03057)

[15] Alexander S. Kechris and Yiannis N. Moschovakis (eds.), Cabal Seminar 76-77, Lecture Notes in Mathematics, vol. 689, Springer, Berlin, 1978. MR526912 (80b:03004)

[16] A. H. Lachlan, Uniform enumeration operations, J. Symbolic Logic 40 (1975), no. 3, 401-409. MR0379156 (52 \#62)

[17] Andrew Lewis, On a question of Sacks a partial solution on the positive side, New computational paradigms, 2005, pp. 15-63.

[18] Andrew S. Marks, Recursion theory and countable Borel equivalence relations, Ph.D. Thesis, 2012.

[19] Donald A. Martin, The axiom of determinateness and reduction principles in the analytical hierarchy, Bull. Amer. Math. Soc. 74 (1968), 687-689. MR0227022 (37 \#2607)

[20] Antonio Montalbán, Embedding jump upper semilattices into the Turing degrees, J. Symbolic Logic 68 (2003), no. 3, 989-1014. MR2000490 (2004h:03089)

[21] Gerald E. Sacks, Degrees of unsolvability, 2nd edition, Princeton University Press, Princeton, N.J., 1966.

[22] - Higher recursion theory, Perspectives in Mathematical Logic, Springer-Verlag, Berlin, 1990. MR1080970 (92a:03062)

[23] Richard A. Shore and Theodore A. Slaman, Defining the Turing jump, Math. Res. Lett. 6 (1999), no. 5-6, 711-722. MR1739227 (2000m:03104)

[24] Theodore A. Slaman, Aspects of the Turing jump, Logic Colloquium 2000, 2005, pp. 365-382. MR2143887 (2006b:03049)

[25] Theodore A. Slaman and John R. Steel, Definable functions on degrees, Cabal Seminar 81-85, 1988, pp. 37-55. MR960895 (89m:03033)

[26] John R. Steel, A classification of jump operators, J. Symbolic Logic 47 (1982), no. 2, 347358. MR654792 (84i:03085)

[27] Simon Thomas, Universal Borel actions of countable groups. Accepted for publication in Groups, Geometry, and Dynamics.

[28] _ Some applications of superrigidity to Borel equivalence relations, Set theory (Piscataway, NJ, 1999), 2002, pp. 129-134. MR1903855 (2003j:03061)

[29] _ Continuous versus Borel reductions, Arch. Math. Logic 48 (2009), no. 8, 761-770. MR2563816 (2011c:03105)

[30] _ Martin's conjecture and strong ergodicity, Arch. Math. Logic 48 (2009), no. 8, 749759. MR2563815 (2011g:03120)

[31] _ Popa superrigidity and countable Borel equivalence relations, Ann. Pure Appl. Logic 158 (2009), no. 3, 175-189. MR2500091 (2009m:03074)

[32] Benjamin Weiss, Measurable dynamics, Conference in modern analysis and probability (New Haven, Conn., 1982), 1984, pp. 395-421. MR737417 (85j:28027)

[33] W. Hugh Woodin, A tt version of the Posner-Robinson theorem, Computational prospects of infinity. Part II. Presented talks, 2008, pp. 355-392. MR2449474 (2009j:03067)

[34] Robert J. Zimmer, Ergodic theory and semisimple groups, Monographs in Mathematics, vol. 81, Birkhäuser Verlag, Basel, 1984. MR776417 (86j:22014) 
Department of Mathematics, California Institute of Technology

E-mail address: marks@caltech.edu

Department of Mathematics, University of California at Berkeley

E-mail address: slaman@math.berkeley.edu

Department of Mathematics, University of California at Berkeley

E-mail address: steel@math.berkeley.edu 